\title{
Retrieval of Offline Handwritten Signatures
}

\author{
H.N. Prakash \\ Department of Studies in Computer Science, \\ University of Mysore, \\ Manasagangothri, Mysore-570 006, India
}

\author{
D. S. Guru \\ Department of Studies in Computer Science \\ University of Mysore \\ Manasagangothri, Mysore-570 006, India
}

\begin{abstract}
Similarity retrieval of images is an important task in database applications. In such applications, effective organization and retrieval of images can be achieved through indexing. In this paper, the problem of quick retrieval of offline signatures in the context of database of signature images is addressed. The proposed methodology retrieves signatures in the database of signature images for a given query signature according to the decreasing order of their spatial similarity with the query. Similarity computed is based on orientations of corresponding edges drawn in between geometric centers (centroids) of the signature image. We retrieve the best hypotheses in a simple yet efficient way to speed up the subsequent robust recognition stage. The runtime of the signature recognition process is reduced, because the scanning of the entire database for a given query is narrowed down to comparing the query with a few top retrieved hypotheses. The experimentation conducted on a large MCYT_signature database [1] has shown promising results. The results demonstrate the efficacy of the proposed methodology.
\end{abstract}

\section{Keywords}

Signature retrieval; Spatial similarity; Offline signature.

\section{INTRODUCTION}

A lot of research has been carried in the field of handwritten signature over the last two decades and a several verification and recognition models have appeared in the literature. There are two different categories in handwritten signatures: Offline (static) signatures and Online (dynamic) signatures. Offline signature is nothing but an image of a signature image captured by a camera or obtained by scanning a signature, which is on a paper or a document. Offline signatures (conventional signatures) are supplemented by other features like azimuth, elevation and pressure in case of online signatures. Online signatures are more robust as they store additional features, other than just signature image like azimuth, elevation and pressure in case of online signatures. Handwritten signature is one of the commonly used biometrics for general authentication in almost all transactions. Generally, any biometric identification problem [2] has two distinct phases: i) recognition and ii) verification. In verification, the query signature is contrasted with a limited set of signatures of the class whose identity is claimed. At the recognition phase, presence of an identity in the database is ascertained [3]. It involves matching stage that extends to entire dataset/database, which is more time consuming.
Research on online signature verification is wide spread while those on offline are not many. Again in both the cases, the research on signature verification is wide spread compared to the research on signature recognition. Both signature verification and signature recognition has distinct applications. Signature verification is an active research field with application like validation of checks and other financial documents. Due to practical significance of signature verification a lot of research has been carried out and many techniques like dynamic time warping [4], Baysian classifiers [5], Neural networks [6], support vector machine [7], Hidden Markov Model [8] have been already recommended [9] investigated spatial properties of handwritten images through matrix analysis. For details of progress in online signature verification, the readers are referred to a review paper [10].

Theoretical point of view signature verification is $1: 1$ matching process while signature recognition is $1: \mathrm{N}$ matching problem hence signature recognition looks more complex. The same techniques used in signature verification can be used here taking into consideration the time complexities and 1: $\mathrm{N}$ matching problem. Signature recognition has potential application like an identification tool. For example, automatic signature recognition system can be used in validation of identity of an individual who needs an access to secured zone or security sensitive facilities [11]. Other potential application of signature recognition is in law-enforcement applications, which requires identification of perpetrators, and in analysis of some historical documents [12].

Some techniques employed in the area of signature recognition are: application of vector quantization - dynamic time warping scheme for signature recognition [13], applications of active deformable models for approximating the external shape of the signatures [14], [15] comparison of support vector machines and Neural Network for signature recognition is made in [15], Comparison of support vector machines and multilayer perceptrons for signature recognition [16].

\subsection{Related Work: Signature Retrieval}

In the work [2] signature recognition and signature verification are treated as two separate consecutive stages, where successful verification is highly dependent on successful recognition. Pavlidis [14] state that it would be of great value if an intelligent signature identification system were capable of arriving at a decision (recognition and verification) based only on the signature of the user. In this context, the signature recognition system is 
applied as an efficient preprocessing stage for signature verification.

Essentially any signature recognition system can be optimized when the query signature is compared with best hypotheses than the entire database. Hence, in this work we focus on quick retrieval of offline signatures for optimizing subsequent robust recognition/verification system. Hence, signature retrieval mechanism that retrieves the best hypotheses from the database attains importance. Efficient retrieval of handwritten signatures is still a challenging work in the situations of a large signature database. Unlike fingerprint, palm print and iris, signatures have significant amount of intra class variations, making the research even more compelling. This approach with the potential applications of signature recognition / verification system optimized with efficient signature retrieval mechanism, justify from our point of view the importance of finding the effective automatic solutions to signature recognition problems.

In so far, the only work on offline signature retrieval is by Han and Sethi [17]. They work on handwritten signatures and use a set of geometrical and topological features to map a signature onto 2D-strings [18]. However, 2D-strings are not invariant to similarity transformations and any retrieval systems based on them are hindered by many bottlenecks [19]. There are several approaches for perceiving spatial relationships such as ninedirectional lower triangular matrix (9DLT) [20] and triangular spatial relationship (TSR) [21] etc. In order to overcome the said problem, in our previous work, we have proposed an online signature retrieval model [22] using global features based on $\mathrm{SIM}_{R}$. In this paper, we propose offline signature retrieval model based on spatial topology of geometric centers, which quickly retrieve the signatures from the database for a given query in the decreasing order of their spatial similarity with the query. Consequently the proposed system can be used as a preprocessing stage which reduces the runtime of the recognition process as scanning of the entire database is narrowed down to comparing the query with a top few retrieved hypotheses. Experimentation has been conducted on a MCYT_signature database [1] and it has shown promising results.

The remaining part of the paper is organized as follows. The proposed methodology is explained in section 2. The details of the experimentations and corresponding results are given in section 3, and finally in section 4 some conclusions are drawn.

\section{PROPOSED MODEL}

This section explains the method of extraction of features and subsequently explains the retrieval model.

\subsection{Feature Extraction}

The geometric centers represent the pixel distribution of the signature image which in turn depends on handwritten signature pattern. In the proposed system signature image is binarized using the histogram based global threshold [23]. Then, we find the geometric centroid of the image and then we split the signature image vertically at the geometric centroid to get two partitions. In the next step, we find the geometric centroids / centers of each partition to split each of the partitions horizontally at their geometric centers. This procedure of finding centers and splitting the partitions at the centers is continued recursively vertically and horizontally in an alternative way till a desired depth of the splitting is reached. Generally we extract $n=\left[(2)^{r}-1\right]$ centers, where $r=1,2,3, \ldots, k$, so that we can have even number splits throughout the signature image, where $r$ is the depth of the splits. The above procedure is continued with recursive vertical and horizontal splits at the geometric centers of the split portions. The above procedure can be started with horizontal split (first split being horizontal instead of vertical split) also but horizontal and vertical splits should take place consecutively. We use only the centers obtained by above procedure with first split being vertical. Centers extracted for each split portions are labeled as $1,2,3, \ldots$, $n$ in sequence as shown in Figure1.

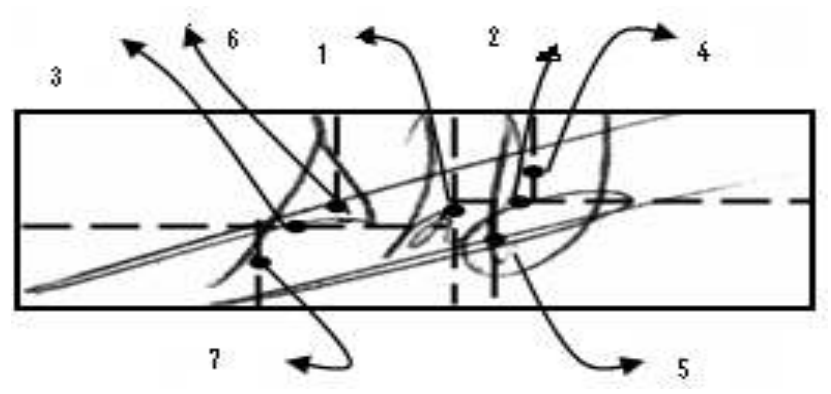

Figure 1. Geometric centers of split portions of a signature image

\subsection{Retrieval Scheme}

Our approach involves extracting geometric centers as explained in previous section 2.1. Say we get ' $n$ ' extracted geometric points by performing vertical split and horizontal split successively. The first geometric center is labeled as ' 1 ' and the second as ' 2 ' and so on and so forth until ' $n$ ', the last geometric point. We illustrate the proposed methodology with $n=5$ points for the sake of clarity even though we extract $n=\left[(2)^{r}-1\right] ; r=1,2, \ldots, k$. points so that we can have uniform splits throughout the signature image.

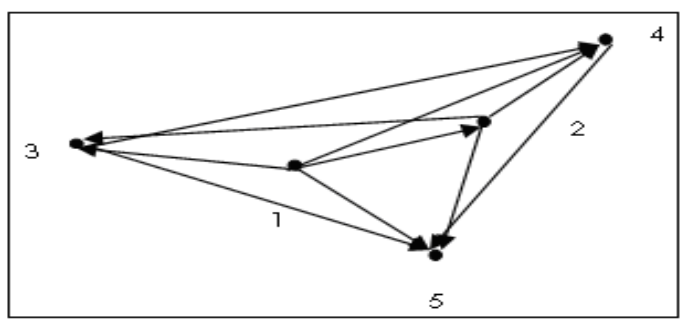

Figure 2. Geometric centers with labels as nodes and edges joining various nodes

A directed graph of ' $n$ ' geometric centers is envisaged where directions originate from the node with smaller label to the one with larger label as shown in Figure 2 for $n=5$. A vector $V$ consisting of the slopes of all the directed edges form the symbolic representation of a signature and is given by 


$$
V=\theta_{12}, \theta_{13}, \ldots, \theta_{1 n}, \theta_{23}, \theta_{24}, \ldots, \theta_{i j}, \ldots, \theta_{n-1 n}
$$

where $\theta_{i j}$ is the slope of the edge directed from node $i$ to node $j$,

$$
1 \leq i \leq n-1, \quad 2 \leq j \leq n, \text { and } i<j .
$$

Let $S_{1}$ and $S_{2}$ be two signatures, V1 and $V_{2}$ be the corresponding vectors representing the slopes of the edges in $S_{1}$ and $S_{2}$. Now the similarity between $S_{1}$ and $S_{2}$ is analogous to the similarity between the vectors $V_{l}$ and $V_{2}$. Let

$$
\begin{aligned}
& V_{1}={ }^{S_{1}} \theta_{12},{ }^{S_{1}} \theta_{13}, \ldots,{ }^{S_{1}} \theta_{1 n},{ }^{S_{1}} \theta_{23},{ }^{S_{1}} \theta_{24}, \ldots,{ }^{S_{1}} \theta_{i j}, \ldots,{ }^{S_{1}} \theta_{n-1 n} \\
& V_{2}={ }^{S_{2}} \theta_{12},{ }^{S_{2}} \theta_{13}, \ldots,{ }^{S_{2}} \theta_{1 n},{ }^{S_{2}} \theta_{23},{ }^{S_{2}} \theta_{24}, \ldots,{ }^{S_{2}} \theta_{i j}, \ldots,{ }^{S_{2}} \theta_{n-1 n}
\end{aligned}
$$

Let $\Delta V=\left|V_{1}-V_{2}\right|$. That is,

$$
\Delta V=\Delta \theta_{12}, \Delta \theta_{13}, \ldots, \Delta \theta_{1 n}, \Delta \theta_{23}, \Delta \theta_{24}, \ldots, \Delta \theta_{i j}, \ldots, \Delta \theta_{n-1 n}
$$

Here the $\Delta V$ represents the vector of the absolute differences in the slopes of corresponding edges in signatures $S_{l}$ and $S_{2}$. The total number of edges is $(n(n-1)) / 2$. Assuming a maximum possible similarity of 100 , each edge contributes a value of $100.00 /(n(n-1) / 2)$ towards the similarity. If the difference in the corresponding edge orientations of the two signatures is zero then the computed similarity value is maximum. When the differences in corresponding edge orientations tend to be away from zero, then the similarity between the two signatures reduces. In this case contribution factor [24] towards similarity from each corresponding edges directed from node $i$ to node $j$ in $S_{l}$ and $S_{2}$ is

$$
\frac{100.00}{n(n-1) / 2}\left(\frac{1+\cos \left(\Delta \theta_{i j}\right)}{2}\right)
$$

where $\Delta \theta_{i j}=\left|{ }^{S_{1}} \theta_{i j}-{ }^{S_{2}} \theta_{i j}\right|$,

$$
1 \leq i \leq n-1, \quad 2 \leq j \leq n \text {, and } i<j .
$$

Consequently the similarity [20] between $S_{1}$ and $S_{2}$ due to all edges is

$$
\operatorname{SIM}\left(S_{1}, S_{2}\right)=\frac{100.00}{n(n-1) / 2} \sum_{i j}\left(\frac{1+\cos \left(\Delta \theta_{i j}\right)}{2}\right) .
$$

where $1 \leq i \leq n-1, \quad 2 \leq j \leq n$, and $i<j$.

Rotation invariance is achieved by aligning the first edge of the query signature with that of database signature before comparing. The scale normalization is achieved with respect to the largest edge in the signature image. Consequently the proposed method is robust so that it can deal scale and rotation invariance which is common in handwritten signatures. The computation complexity of the proposed methodology is $O\left(n^{2}\right)$. During retrieval, the geometric centers of query signature are extracted and slopes of the edges between all the possible geometric centers are computed to form a query vector. The query feature vector is compared with the training feature vectors in the knowledgebase. The feature vector size of the query and training should be same. Signatures are retrieved according to the similarity ranks and top $K$ retrievals are selected for further matching for accurate recognition / verification.

\section{EXPERIMENTAL RESULTS}

The dataset: The MCYT-75 offline signature corpus [1] consists of 30 signatures; 15 are genuine and remaining 15 are forgeries of the 75 individuals. Totally it forms a signature database of 1125 (i.e. $75 \times 15)$ genuine and 1125 (i.e. $75 \times 15$ ) forged offline signatures. (see Figure. 3)
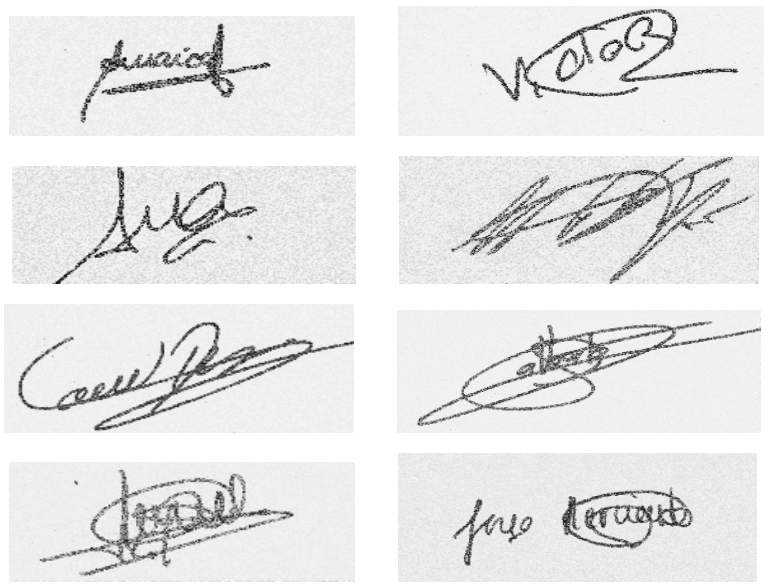

Figure 3. Sample offline signature from $M C Y T_{-}$signature corpus

The comparison of retrieval performances of the proposed method of signature retrieval and the method of signature retrieval based on similarity measure $\operatorname{SIM}_{\mathrm{G}}$ [25] made through a series of extensive experimentation in this section. $\operatorname{SIM}_{\mathrm{G}}$ is a geometry based algorithm for computing similarity between symbolic images. This algorithm is of linear time complexity $O(n)$. For the more details of the similarity measure $\mathrm{SIM}_{\mathrm{G}}$ the readers are refer to [25]. Our interest here is to compare the performance of proposed retrieval method which is of $O\left(n^{2}\right)$ computational complexity with the performance of retrieval method based on $\mathrm{SIM}_{\mathrm{G}}$ which is of linear time complexity $O(n)$.

We have evaluated the retrieval performance for 7 genuine signatures per class as database signatures and remaining 8 signatures per class as query signatures. (see Table 1). In total we have made 315000 comparisons in our experimentations for 525 database and 600 query signatures as shown in Table1 and this shows the efficacy of the system. In all these cases retrieval process is as follows: given a query signature it is matched with all the signatures in the database and the corresponding similarity values are computed. Similarity values are then stored in decreasing order. The top $K$ hypotheses are retrieved. 
Table 1. Query and database signatures combination

\begin{tabular}{|l|c|}
\hline $\begin{array}{l}\text { Number of database signatures (training) per } \\
\text { class }\end{array}$ & 7 \\
\hline $\begin{array}{l}\text { Number of query signatures } \\
\text { per class }\end{array}$ & 8 \\
\hline $\begin{array}{l}\text { Total number of database } \\
\text { signatures (training) }\end{array}$ & $8 \times 75=525$ \\
\hline $\begin{array}{l}\text { Total number of } \\
\text { query signatures }\end{array}$ & $\begin{array}{c}525 \times 600= \\
315000\end{array}$ \\
\hline Total number of signature comparisons & \\
\hline
\end{tabular}

The output of the retrieval system is the top $K$ hypotheses. We define the correct retrieval $(C R)$ for the performance evaluation of retrieval system as

$$
C R=\left(K_{c} / K_{d}\right) \times 100
$$

where $K_{c}$ is the number of correctly retrieved signatures, $K_{d}$ is the number of signatures in the database

Retrieval experiments are conducted for different number of extracted geometric points $n: 7,15$ and 31 . For these set of extracted geometric centers we have evaluated correct retrieval performance against the percentage of database scan for varying number of training samples. It can be observed from Fig. 4 that the retrieval performance is good for 31 geometric centers when compared to 7 and 15 geometric centers irrespective of number of training samples. By the observation of Figure 4 retrieval performance of the proposed method is best when compared to the method based on $\mathrm{SIM}_{\mathrm{G}}$. For the proposed method (see Figure 4(b)) with 31 geometric points, just for 5\% database scan we have $98 \%$ correct retrieval, for $8 \%$ database scan we have $99 \%$ correct retrieval and for $18 \%$ database scan correct retrieval is $100 \%$. Where as for the method based on $\operatorname{SIM}_{\mathrm{G}}$ (see Figure 4(a)) with 31 geometric points, just for 5\% database scan we have $91 \%$ correct retrieval, for $8 \%$ database scan we have $93 \%$ correct retrieval and for $30 \%$ database scan correct retrieval is $100 \%$.

Since retrieval accuracy is an important issue in the case of signature databases, retrieval accuracy is defined in terms of precision and recall rates. The precision rate is defined as the percentage of retrieved signatures which belong to the given

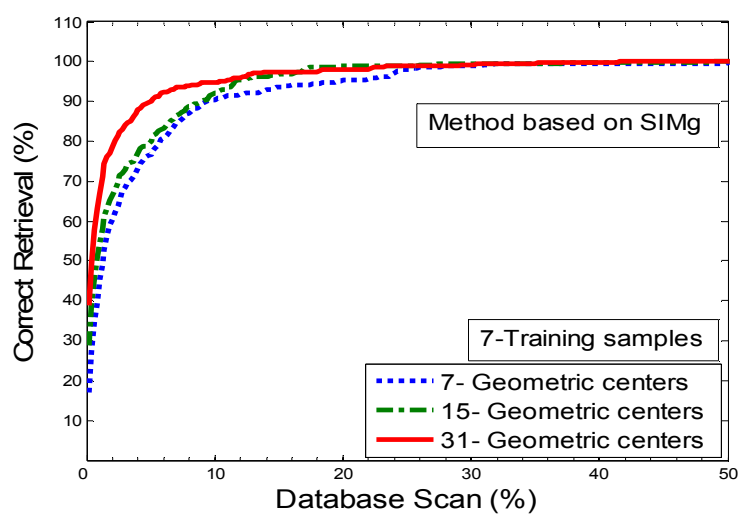

(a)

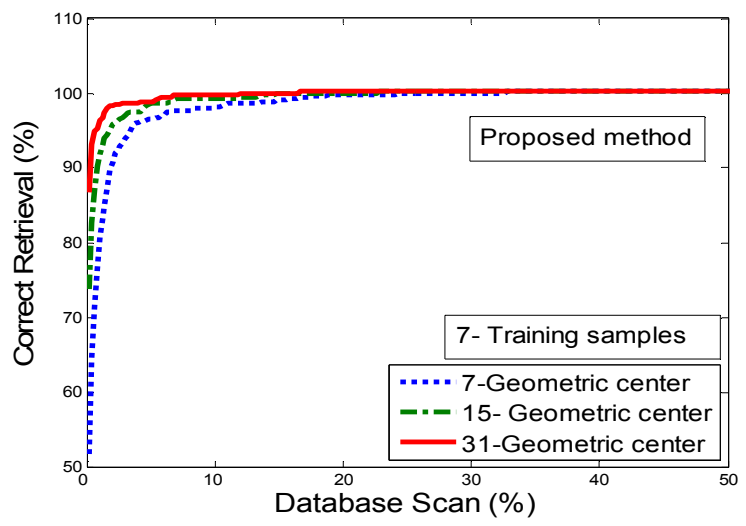

(b)

Figure 4. Retrieval performance with different number of geometric centroids

query class among the total number of retrieved signatures. The recall rate is defined as the percentage of retrieved signatures, which are similar to query signature among the total number of signatures similar to the query signature in the database. It can easily be seen that both precision and recall are the functions of total number of retrieved signatures. Hence, it is desirable to have a system that has both high precision and high recall rates. We have measured precision and recall rates for each query signature by considering the number of signatures retrieved in top $K$ positions. If $K_{l}$ refers to the number of signatures retrieved in top $K$ positions which belong to the query class, $K_{2}$ refers to the number of signatures in the database which belong to query class, then the precision rate is given by $\left(K_{l} / K\right)$ and recall rate is given by $\left(K_{I} / K_{2}\right)$. To evaluate the performance of the proposed methods, we also compute the Precision and Recall ratios. The results are shown in Fig. 5. Proposed method shows very good precision and recall ratios compared to a method based on $\mathrm{SIM}_{\mathrm{g}}$. The best performance (precision) is observed in Fig. 6(b) for the proposed method for 31 geometric center points.

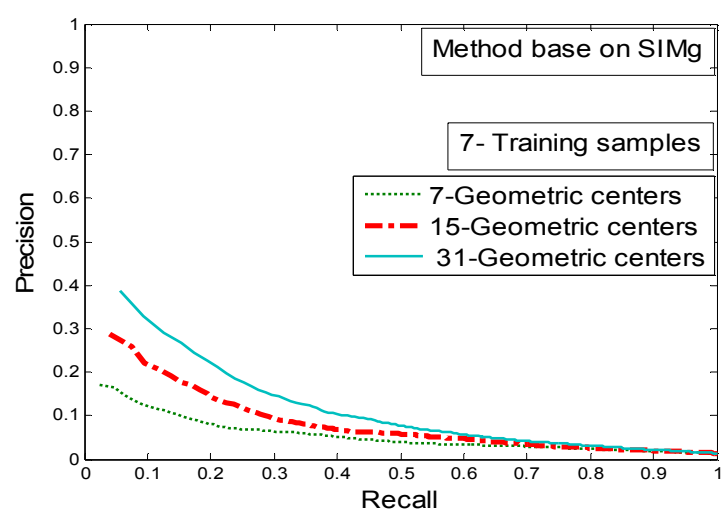

(a) 


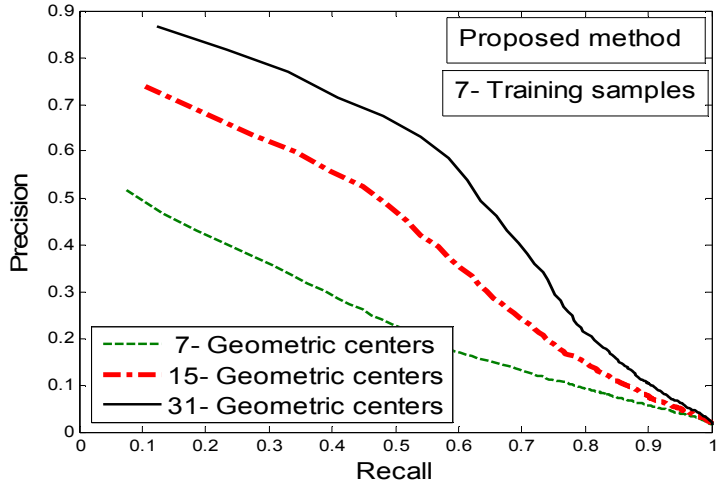

(b)

Figure 5. Precision ratio v/s recall ratio for different number of geometric centroids.

Further experiments are conducted to study the correct retrieval against the variations in similarity threshold (fig.6 (a)). We define the correct retrieval $(C R)$ for the performance evaluation of retrieval system as $C R=\left(K_{c} / K_{d}\right) \times 100$ where $K_{c}$ is the number of correctly retrieved signatures, $K_{d}$ is the number of signatures. For $80 \%$ similarity, we get Correct Retrieval of $15 \%$ and for similarity threshold below 50(\%), we get Correct Retrieval of $100 \%$. Using the binomial prediction model [26] we compute the probability of correct retrieval against the rank from gallery of signatures. The probability that the correct retrieval occurs at rank $r$ is given by the binomial probability distribution. The probability distribution indicates that the correct retrieval begins at minimum rank value $K=5$, which achieves probability of correct retrieval 1 $(100 \%)$ at $\mathrm{K}=120$. This decides the value for the top $\mathrm{K}$ hypothesis. The output of the retrieval system is the top $K$ hypotheses.

\section{CONCLUSION}

Experiments were conducted for quick retrieval of offline signatures and results are presented. The retrieval performance of the proposed method based on edge correspondence is compared with the retrieval method based SIM $_{\mathrm{g}}$. The proposed method is simple, efficient and outperforms the retrieval system based $\mathrm{SIM}_{\mathrm{g}}$ respect to all parameters (Precision, Recall and Correct Retrieval). In the proposed work we used large database of 1125 signature images and further the proposed method is simple when compared to the only work on signature retrieval by Han and Sethi [17]. Because extraction of geometrical and topological features such as loops end points branch points cross points etc used in [17] to map a signature onto 2D-strings is cumbersome and computationally intensive. In [17] only 120 images are used.

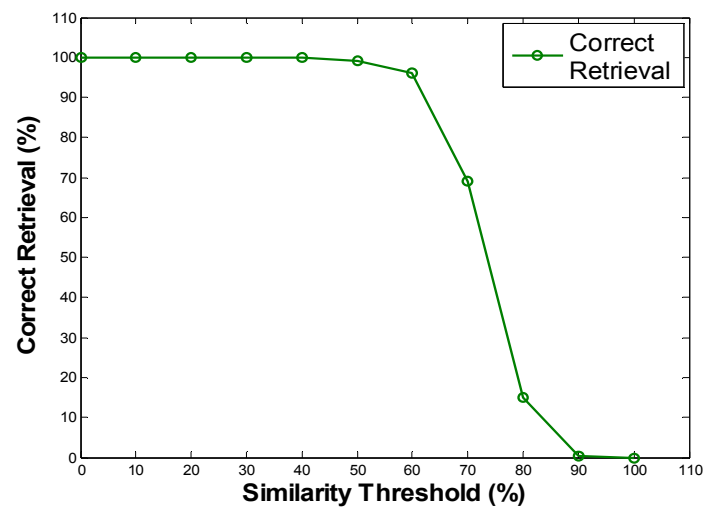

(a)

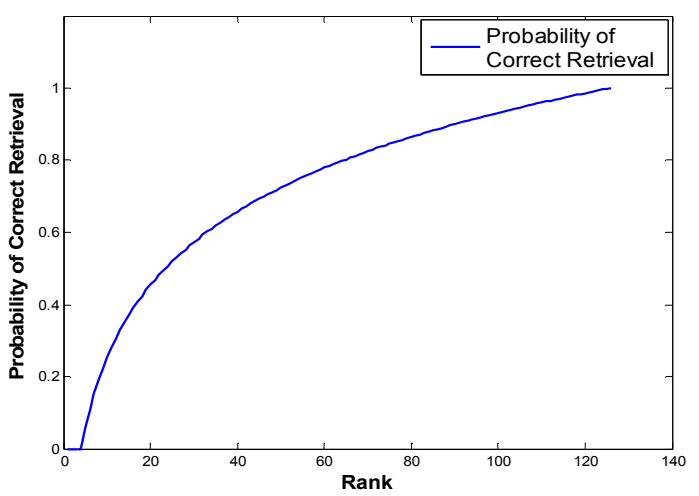

(b)

Fig. 6 (a) Correct Retrieval (\%) versus Threshold. (b) Probability of Correct Retrieval versus Rank

With this large dataset of signatures, we have obtained promising results in retrieving top $K$ hypotheses. We have obtained $98 \%$ correct retrieval for just $5 \%$ data scan and $99 \%$ correct retrieval for $8 \%$ data scan (Fig. 4(b)). The best precision $98 \%$ is observed in Fig. 5(b) for the proposed method for 31 geometric points.

The minimum percentage of database scan required to retrieve relevant signatures for all queries is supposed to be fixed experimentally. This is essentially a $K$-nearest neighbor problem and $K$ best hypotheses should be retrieved. An attempt has been made in the work of Ghosh [27] in this regard where the parameter ' $K$ ' is fixed without experimentation. Hence, the decision of arriving at the optimal percentage of database scan where all the authentic queries find a match can be fixed up analytically.

\section{Acknowledgments}

Authors thank Dr. Julian Firrez Auguilar, Biometric Research Lab-AVTS, Madrid, Spain for providing MCYT_signature dataset 


\section{REFERENCES}

1. http://atvs.ii.uam.es/mcyt100s.html.

2. Ismail M.A., and Gad S., 2002. "Offline Arabic signature verification”, Pattern Recognition, vol. 33, pp. $1727-1740$

3. Lee S and Pan J. C., 1992. "Offline tracing and representation of signatures", IEEE Transaction Systems Man and Cybernetics, vol. 22, pp. 755-771.

4. Fang P., Zhang Cheng Wu, Fei Shen, Yun Jian Ge and Bing Fang, 2005. "Improved DTW algorithm for signature verification based on writing forces", International Conference on Intelligent Computing, LNCS 3644, pp.631-640.

5. Xiao X. and Graham Leedham, 2002. "Signature verification using a modified Bayesian network", Pattern Recognition, vol. 35, pp. 983-995.

6. Bajaj R. and Chaudhury S., 1997. "Signature using Multiple neural Classifiers", Pattern Recognition, vol. 30 , pp. 1-7.

7. Ji Hong-Wei and Zhong-Hua Quan, 2005. "Signature verification using wavelet transform and support vector machine", \{ \it International Conference on Intelligent Computing (ICIC-2005), LNCS 3644, pp. 671-678.

8. Kashi R., .Hu. W.L. Nelson, W. Turin, 1998. "A Hidden Markov Model approach to online handwritten signature verification", International Journal of Document Analysis and Recognition (IJDAR), vol. 1, pp. 102-109.

9. Found B., Rogers D. and Schmittat R., 1998 “ Matrix Analysis : A technique to investigate the spatial properties of handwritten images, Journal of Forensic Document Examination, vol. 11, pp. $54-74$.

10. Dimauro G.,S. Impedovo, M. G. Lucchese, R. Modugno and G. Pirlo, 2004. Recent Advancement in Automatic Signature Verification. Proceedings of $9^{\text {th }}$ International Workshop on Frontiers in Handwriting Recognition (IWFHR-9), pp. 179-184.

11. Lee S., and Pan J.C., 1992. "Offline tracing and representation of signatures", IEEE Transactions, Systems Man and Cybernetics, vol. 22, pp. 755-771.

12. Ismail M.A., and Gad S., 2000. "Offline Arabic signature verification", t Pattern Recognition, vol.33, pp. 1727-1740.

13. Marcos Foundez- Zanuy, 2006. "Online signature recognition based on VQ_DTW", Pattern Recognition, vol. 40, issue 3, pp 981-992.

14. Pavlidis I.,Papanikolopouls N. P. and Mavuduru R., 1998. "Signature identification through the use of deformable structures", \{lit Signal processing\}, vol. 71, pp. 187-201.
15. Leclerc and Plomondon, 1997. "Automatic signature verification: the state of the art", International Journal of Pattern recognition and Artificial Intelligence\}, vol. 8, pp. 643-660.

16. Martinez E. F., Sanchez A. and Velez J., 2006. "Support vector machines versus Multilayer perceptrons for efficient offline signature recognition", Artificial Intelligence, vol. 19, pp. 693-704.

17. Han Ke and Sethi I. K., 1995. "Handwritten signature retrieval and identification", Pattern Recognition Letters, vol. 17, pp. 83-90.

18. Chang S. K. and Li Y., 1998. "Representation of multi resolution symbolic and binary pictures using 2D-H strings", Proceedings of the IEEE Workshop on Languages for Automata, Maryland, pp.190-195.

19. Guru D. S., Punitha P and Nagabhushan P., 2003. "Archival and retrieval of symbolic images: An invariant scheme based on triangular spatial relationship", Pattern Recognition Letters, vol. 24, No. 14, pp. 2397-2408.

20. Chang C. C., 1991. "Spatial match retrieval of symbolic pictures", Information Science and Engineering, vol. 7, No. 3, pp. 405-422.

21. Guru D. S and Nagabhushan P. 2001. "Triangular spatial relationship: A new approach for spatial knowledge representation", Pattern Recognition Letters, vol. 22, No. 9, pp. 999-1006.

22. Guru D. S., H. N. Prakash and T. N. Vikram 2007. "Spatial topology of equitemporal points on signature for retrieval", in proc. International conference on Pattern Recognition and Machine Intelligence (PReMI2007), Kolkata, India, LNCS 4815, pp.128-135.

23. Otsu N., 1994, A threshold selection method from grey level histogram. IEEE Transactions on Systems, Man and Cybernetics, Vol. 9, pp.62-66.

24. Gudivada V. N and Raghavan V. V., 1995. "Design and evaluation of algorithms for image retrieval by spatial similarity", ACM Transactions on Information Systems, vol. 13, No. 2, pp. 115-144.

25. Gudivada V. N., 1998. " $\Theta R$-string: a geometry-based representation for efficient and effective retrieval of images by spatial similarity", IEEE trans. Knowledge Data Engineering, vol. 10(3), pp 504-512.

26. .Rong Wang and Bir Bhanu, 2007. Predicting fingerprint biometrics performances from small gallery, Pattern recognition letters, vol. 28 (1). pp. 40-48.

27. Ghosh A. K. 2006. "An optimum choice of $k$ in nearest neighbor classification", Computational Statistics and Data Analysis, vol. 50, issue 11, pp. 3113-3123. 